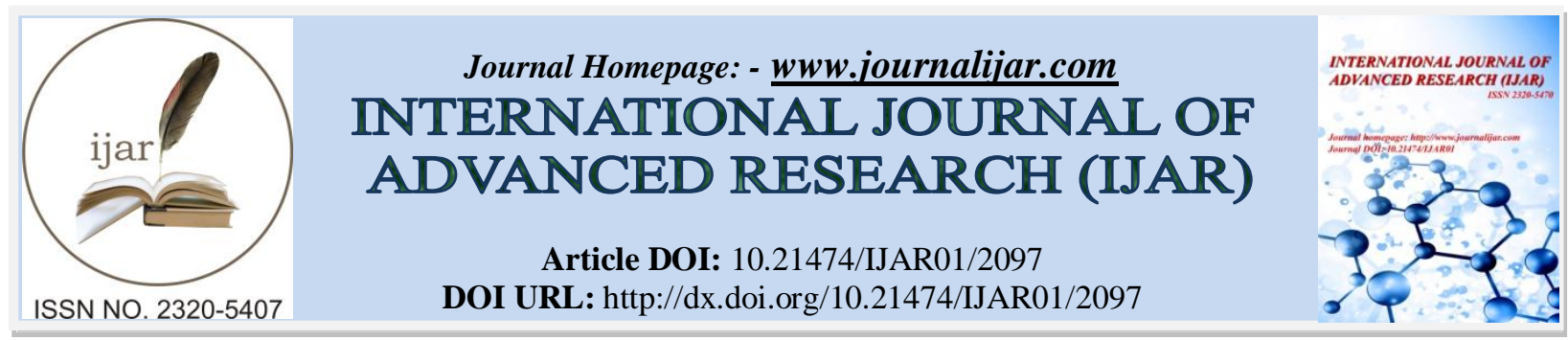

RESEARCH ARTICLE

\title{
A COGITATION ON HYPERGLYCEMIA INDUCED DIABETIC MACULAR EDEMA.
}

Ayesha Habeeb* and Umair Wahedi

Deccan school of pharmacy, Department of pharmacy practice, Dar us salam, Aghapura, Nampally, Hyderabad, Telangana, India.

\section{Manuscript Info}

Manuscript History

Received: 24 September 2016

Final Accepted: 26 October 2016

Published: November 2016

Key words:-

Diabetic retinopathy, Laser photocoagulation, Anti- vascular endothelial growth factor, Optical coherence tomography, Fluorescein angiography, Macular edema.

\begin{abstract}
Diabetic macular edema (DME) is the leading cause of blindness in the diabetic population, and its prevalence is variable. Diabetes mellitus is the leading cause of preventable blindness owing to both diabetic macular edema and complications of proliferative diabetic retinopathy (NDIC 2011). As the incidence of diabetes increases yearly, so does the frequency of vision threatening diabetic retinopathy. Over the last decade, the gold standard for treatment has transitioned from laser photocoagulation to anti- vascular endothelial growth factor agents. With further understanding of the pathophysiology of this disease and the Role of Vascular endothelial cell growth factor(VEGF) in breakdown of endothelial cell-cell junction has been explained clearly. With the aid of advanced imaging modalities such as optical coherence tomography and fluorescein angiography, several novel treatment options lie on the horizon. An understanding of options for management of macular edema is essential for providers and patients alike to help achieve best potential outcomes. For people with diabetes macular edema is the most common cause of vision loss. Both type 1 and type 2 DM patients are susceptible to DME. The likelihood of developing DME increases with severity of retinopathy as well as duration since onset of diabetes. A case report has been presented for treatment approaches in managing suboptimal responses associated with Diabetic Macular Edema(DME)
\end{abstract}

Copy Right, IJAR, 2016,. All rights reserved.

\section{Introduction:-}

The World Health Organization (WHO) estimates that more than 180 million people worldwide have diabetes, and this number is expected to increase and to rise to epidemic proportions within the next 20 years ${ }^{[1]}$. Diabetic retinopathy, one of the most frequent complications of diabetes, remains a major public health problem with significant socioeconomic implications, affecting approximately 50\% of diabetic subjects, and remains the leading cause of blindness in working-age populations of industrialized countries. Diabetic macular edema (DME) is the largest cause of visual acuity loss in diabetes ${ }^{[2]}$. Diabetic macular edema occurs when the macula swells or thickens. The macula is the in the center of the retina and is responsible for your most precise vision. It allows you to read small print and thread needles. The retina acts like the film of the eye. It captures images coming in from the front of the eye and sends them to the brain to see. When the macula is swollen, it does not work properly. This may result in vision loss.

Corresponding Author:- Ayesha Habeeb.

Address:- Deccan school of pharmacy, Department of pharmacy practice, Dar us salam, Aghapura, 


\section{Understanding Macular Edema}
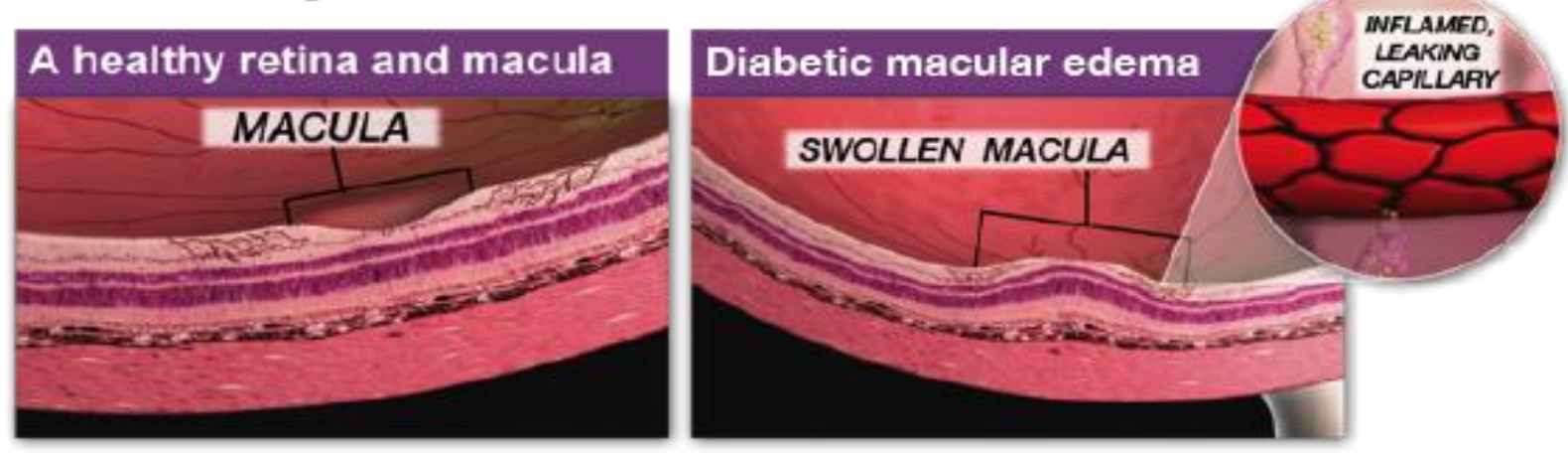

\section{Incidence And Prevelance Of DME:-}

The incidence and prevalence of DME have been reported in different epidemiologic studies with significant variations, depending on the type (type I or II), treatment (insulin, oral hypoglycemic agents, or diet only), and the mean diabetes duration. Although DME can develop at any stage of DR, it is frequently related with increase in duration and severity of DR. DME prevalence, indicated in the Wisconsin Epidemiologic Study in Diabetic Retinopathy (WESDR), is only about $3 \%$ in mild nonproliferative diabetic retinopathy (NPDR), but increases to $38 \%$ in moderate to severe NPDR and to $71 \%$ in eyes with proliferative diabetic retinopathy (PDR). In this study, the incidence of clinically significant DME was $4.3 \%$ in type I diabetic patients and $5.1 \%$ in type II with insulin and $1.3 \%$ in those without insulin. At 10 years, the rate of developing DME was $20.1 \%$ in patients with diabetes type I and $25.4 \%$ in type II diabetic patients needing insulin and $13.9 \%$ in those without insulin ${ }^{[4]}$

\section{Etiology:-}

Prolonged diabetes can damage the tiny blood vessels in the retina that nourish the eye. (This is known as diabetic retinopathy.) These damaged blood vessels may leak blood or other fluid into the eye. This leaking fluid can cause the macula to swell, leading to macular edema.

\section{Pathophysiology:-}

Macula lutea or yellow spot is oval shape pigmented area at the center of retina .Center of Macula lutea is formed by Fovea centralis(fig 1) which is abundant in cone cells and no rod cell and is responsible for central, high resolution, color vision

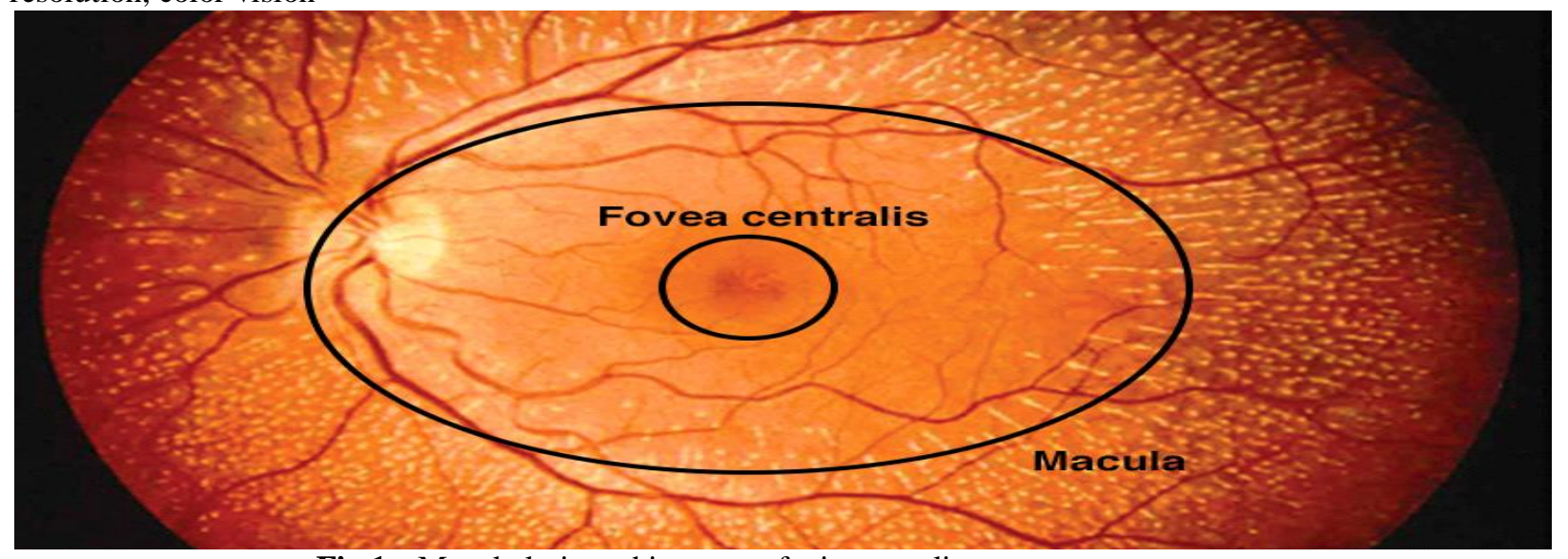

Fig 1:- Macula lutia and its center fovia centralis

Diabetic macular edema is defined as retinal thickening of center of the macula due to increase in volume of retinal tissue water. This thickening of macula can initially be intracellular or extracellular. If it's intracellular (within the cell) it's called cytotoxic edema which is due to alteration in ion exchange which lead to excess of $\mathrm{Na}+$ inside the cell and if it's extracellular (accumulation of fluid in interstitial space) it's called vasogenic edema in this there is 
extracellular fluid accumulation it depends on various factors like blood pressure, blood retinal barrier(BRB) permeability, retinal cell damage, retinal tissue osmotic pressure, retinal tissue compliance, in this situation starlings law is applied which states that fluid movement across membrane of tissue depends on the balance between hydrostatic pressure and oncotic pressure gradient across the membrane in case of macular edema it's disruption blood retinal barrier(BRB). Hydrostatic pressure(HP) gradient is the difference between hydrostatic pressure of the blood in vasculature and hydrostatic pressure in the intraocular tissue i.e. retina this hydrostatic.

Pressure gradient established pushes water from vasculature into tissue interstitial space causing edema. oncotic pressure(OP) is colloidal osmotic pressure cause by proteins mostly albumin in the blood OP is opposite to HP and tends to pull water inside vasculature as OP gradient is difference in OP in the vasculature and OP in the tissue if $\mathrm{OP}$ is less in the vasculature water move into the tissue causing edema. In normal situation HP and OP gradient respectively are equal and opposite so there is no movement of water between tissue and vasculature. Starlings law help in understanding the pathophysiology behind edema in any tissue in retina and can be use in designing treatment.

Hyperglycemia is the major risk factor for the development of diabetic retinopathy (DR) during its progression at any time it may leads to DME. Hallmark of DR is the changes in structure and composition of microvasculature. The exact mechanism associated with DR/DME is not clear. Hyperglycemia induced DR is related to 4 major pathway ${ }^{5}$ polyol pathway ${ }^{6}$ advanced glycation end product pathway ${ }^{7}$ protein kinase C (PKC) ${ }^{8}$ hexosamine pathway. All these pathways cause oxidative stress, inflammation, and vascular dysfunction. Cytokinin and growth factors which leads to vasogenic edema by breaking down of BRB is released due to oxidative stress and inflammation the are VEGF, angioproteins, tumor necrosis factor (TNF), interleukins (ILs), and matric melloproteinases (MMPs).

\section{Blood-Retinal Barrier:-}

BRB formation and maintenance is important for proper vision if there is any breakdown in BRB that will result in vision disturbance and if it is severe can lead to vision loss. Retinal vascular structure is unique which provide necessary nutrients while minimizing interference with light sensing. In addition to unique vasculature retina also contains epithelial barrier (the retinal pigment epithelium) which controls the exchange of nutrient with the choroid vessels. BRB is formed by both vascular endothelium and pigment epithelium they both posses well develop complex tight junction that has high degree of control over solute and fluid permeability. Vascular endothelium and pigment epithelium are called inner BRB and outer BRB respectively. If the is any breakdown in inner BRB that will result in increase in permeability which result in accumulation of plasma protein that leads to high oncotic pressure in neural interstitium which causes vasogenic edema in macula. Tightness of inner BRB is due to coherence of endothelial cell-cell junction, normal basement membrane, pericyte in the outer wall. In diabetes three important alterations occur i.e., (1) breakdown of endothelial cell-cell junction (2) thickening of basement membrane. 


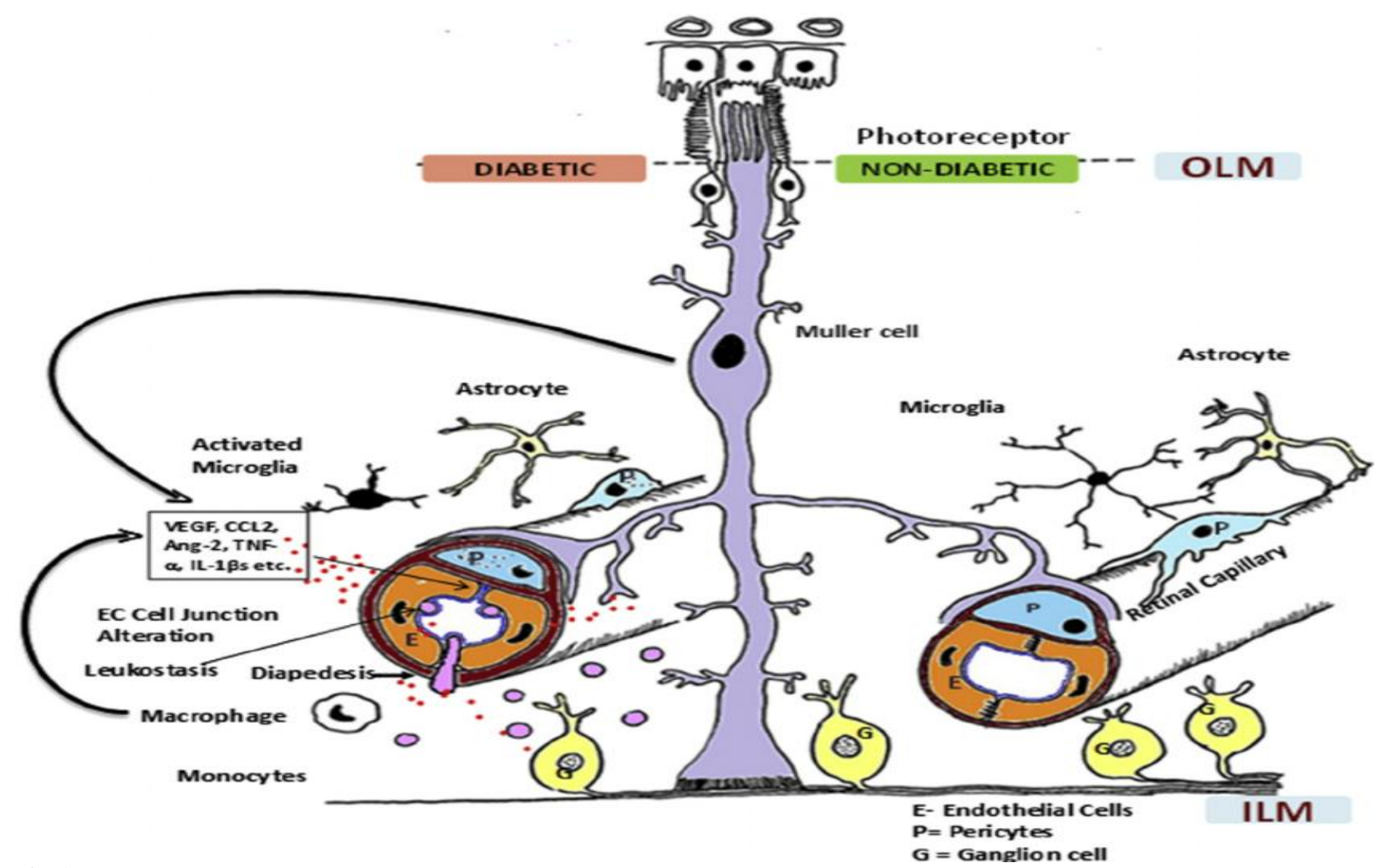

Fig 2:- Neurovascular unit of the retina in nondiabetic and diabetic conditions. Normally, pericytes and endothelial cells constitute the blood retinal barrier (BRB) (retinal capillary) that is covered intimately by multiple processes of the Muller cells (right). Astrocytes and microglia with long processes also surround the capillaries, maintaining the normal homeostasis for neuronal signaling and synaptic transmission. In diabetes, chronic inflammation sets in, contributing to the breakdown of the BRB (left). Müller cells and endothelial cells produce chemokines (including monocyte chemoattractant protein-1) that lead to increased leukostasis, diapedesis, and influx of monocytes into the retina and increased production of cytokines, including vascular endothelial growth factor (VEGF), tumor necrosis factor (TNF)a, interleukin (IL)-1b, matrix metalloproteinase, and angiopoietin (Ang)-2. These inflammatory mediators then result in the breakdown of endothelial cell-cell junctions. Microglia become activated, and there is increased apoptosis of ganglion cells and amacrine cells, which deranges synaptic degeneration. In retinal capillaries, pericyte dropout and thickening of the basement membrane also occur as a result of hyperglycemia, all contributing to increased leakage from vessels. Photoreceptors contribute to production of superoxide and inflammatory proteins in this process. CCL-2 1/4 chemokine ligand 2; EC 1/4 endothelial cell; ILM 1/4 inner limiting membrane; OLM $1 / 4$ outer limiting membrane. ${ }^{12}$
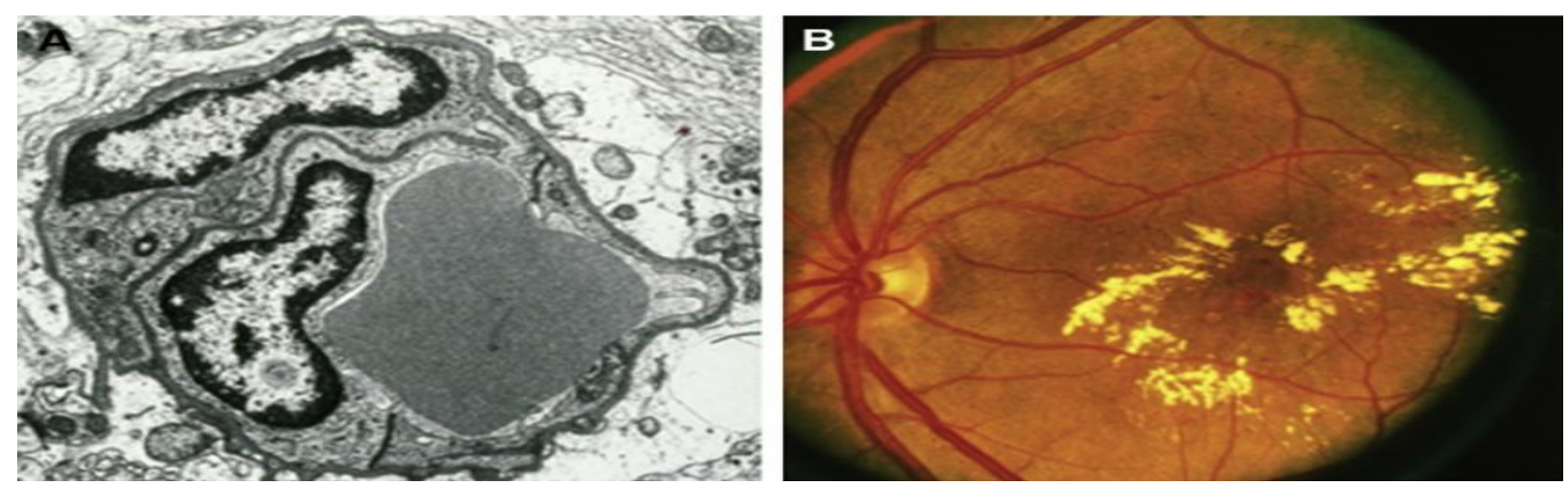

Fig 3 :- Blood retinal barrier alteration in diabetes. A, Electron microscopic view of a monkey retinal capillary showing endothelial cells joined by tight junctions and surrounded by pericytes and a thin basement membrane between cells. B, Fundus photograph of a diabetic patient showing microaneurysms, retinal hemorrhages, and hard 
exudates in the macula. There is also exudation of plasma in the macula, leading to clinically significant macular edema $^{12}$

Breakdown of Endothelial cells-cell junction:- Endothelial cells lining the vessel wall is connected by adherent , tight, gap junctions .Endothelium form monolayer of endothelial cells ${ }^{12}$. These junction complex maintain tissue integrity, barrier function and cell-cell communication respectively(fig ). These endothelial cells adhere to one another through these junctional structure which are formed by transmembrane adhesive proteins that are responsible for homophilic cell-cell adhersion ${ }^{13}$.

BRB specifically contain tight junction (TJ) transmembrane protein occludine whose function is to increase TJ barrier and it increases with increse in pericyte derived angiopoietin-1 .Decrease in occlutin along with increase in para cellular permeability cause by VEGF treated retinal endothelial cell which follows proteolytic mechanism depending on urokinase plasminogen activator ${ }^{[16,17]}$ explains that decrease in occlutin can lead to decrease vascular permeability,Claudin is also trans membrane protein of TJ whose function include maintaining para cellular barrier and regulate para cellular flux which is influx of solute lide $\mathrm{Na}^{+}$or $\mathrm{Cl}^{-[20,21]}$.other TJ associated protein are zona occludin(ZO-1) which is member of membrane associated guanylate kinase homologes(MAGUKs) that are positioned on cytoplasmic surface of junction contact. Adherant junctions(AJ) transmembrane protein cadherin-5 which is endothelial specific cadherin which plays important role in vascular permeability. junctional adhesion molecule(JAM) belong to immunoglobin super family and are located close to tight junction stands ${ }^{[18,19]}$.JAM function is to interact with tight junctions associated proteins to regulate barrier function ${ }^{[22]}$.JAM also interact with leukocytes which mediates inflammatory response ${ }^{[23,24]}$.

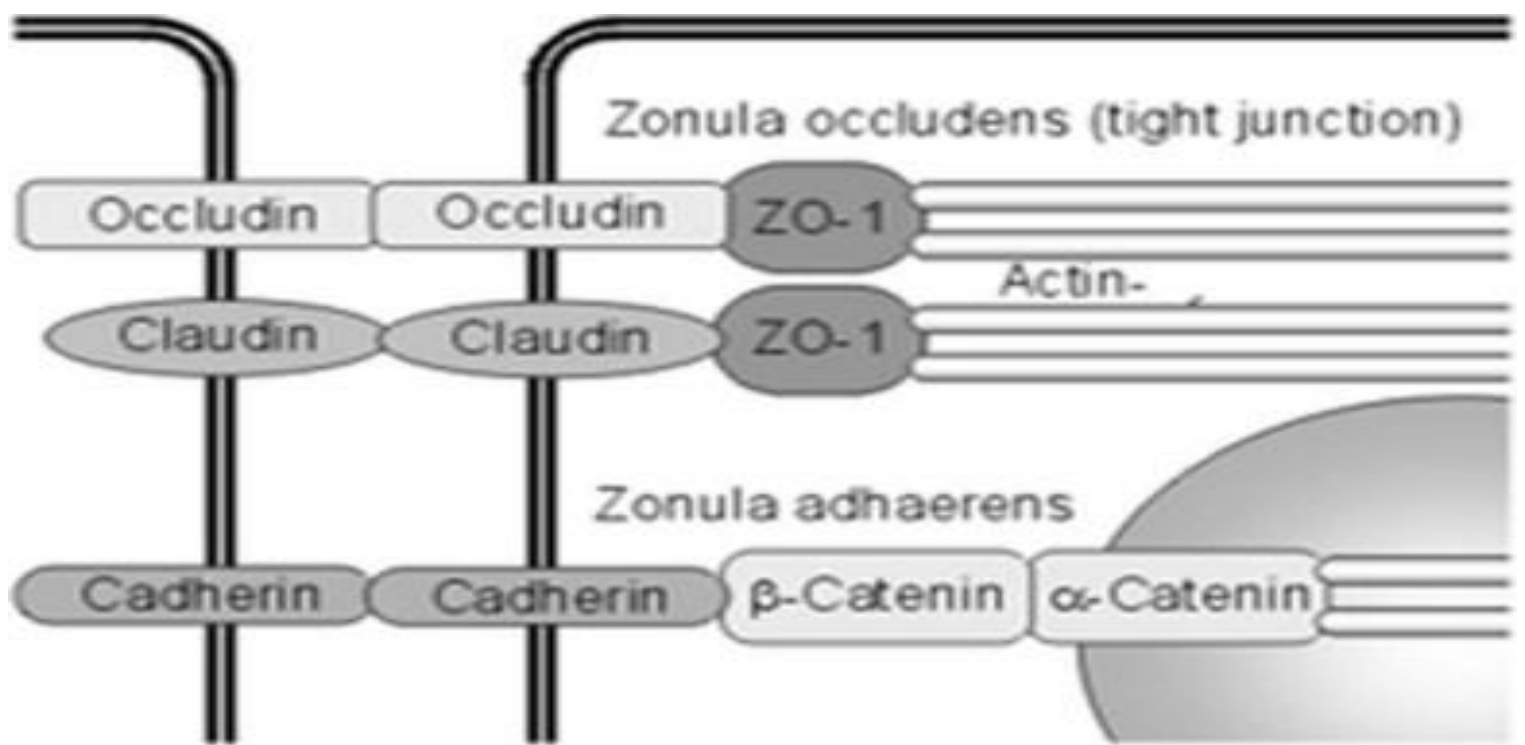

Fig 4:- Intercellular junctions in endothelial cells. Endothelial cells are connected and communicate with each other by tight junctions and adherens junctions. Tight junctions resemble a major part of the inner blood-retinal barrier.

They are built by different proteins, including occludin, ZO-1, and the claudin family ${ }^{[34]}$.

Role of Vascular endothelial cell growth factor(VEGF) in breakdown of endothelial cell-cell junction:-

Introduction:- VEGF is a signaling protein which promotes growth of blood vessels from existing vessels (angiogenesis) and formation of new vessels in embryo(vasculogenesis).It forms a part of mechanism that restores the blood supply during hypoxic condition. It also plays a role in angiogenesis during injury and muscle growth after exercise $^{[25]}$. One of the most important growth and survival factors for endothelium is VEGF ${ }^{[26]}$ VEGF is secreted by different type of cells like Macrophages, keratinocytes, tumour cells, platelets, mesangial cells in kidney, retinal pigmented epithelial cells, pericytes, astrocytes, Muller cells, glial cells, and endothelial cells ${ }^{[25,30]}$. VEGF is heparinbinded glycoprotein that is secreted as homodimer of $45 \mathrm{KDa}^{[27]}$. Over expression of VEFG causes vascular disease of eye, diabetic retinopathy. 
Members of VEGF:- VEFG contains many members which includes VEGF-A, VEGF-B, VEGF-C, VEGF-D , and placental growth factor. VEGF-A contains five mRNA splice variant isoforms: 121,145,165,189,206 depending upon amino acid [30]. VEGF-A is also known as vascular permeability growth factor because it increases vascular permeability ${ }^{[32]}$.VEGF-A also causes vasodilatation by stimulation of nitric oxide synthesis in endothelial cells ${ }^{[29]}$. An alternate inhibitory splice variant of VEFG-A at exon 8 is known as VEGF165b ${ }^{[31]}$ it was detected principally in normal vitreous but not in diabetic vitreous. VEGF165b is endogenous inhibitor of VEGF-A which is about $65 \%$ in normal vitreous ${ }^{[32]}$.

Receptors:- VEGF receptors are of three sub types they are VEGFR 1, VEGFR 2, VEGFR 3. All the members of VEGF family stimulate cellular response by binding to tyrosine kinase receptors i.e VEFGRs . VEGF binds to tyrosine kinase receptors which is present on cell surface and made up of extracellular portion which contain 7 immunoglobin like domain, trans membrane spinning portion and intracellular portion containing split tyrosine kinase domain VEGF by binding undergoes dimerization and transphosphorylation for activation ${ }^{[21]}$. VEGF-A receptor 1(fms like tyrosine kinase-1,FLT-1)and VEGF-A receptor-2 (fetal liver kinase-1,FLK-1) both is activated by VEGF-A .FLK-1 is recognized as vascular permeability factor as one of primary function of FLK-1 is to regulate vessel permeability ${ }^{[29]}$ FLT-1 is negative regulator of FLK-1 third receptor VEGF receptor 3 (fms like tyrosine kinase-4,FLT-4) binds to VEGF-C and VEGF-D.Nuropilin-1 and -2 has also recognized which binds too VEGF165b and VEGF145 respectively.

VEGF and diabetis:- High blood sugar level damages microvasculature of eye which leads to capillary closure and decrease of oxygen due to poor circulation which causes hypoxia and triggers increase in VEFG, angiogenesis . there are many hypothesis proposed regarding VEGF-A and its receptor causing BRB breakdown and diabetic retinopathy development this review article will not be containing those hypothesis ${ }^{[35]}$.

Pericytes:- Pericytes are also called rouget cells or mural cells these cells are embedded in basement membrane where they communicate with endothelial cells of capillaries by physical contact and paracrine signalling ${ }^{[37] .}$ pericytes regulate retinal capillary blood flow and control endothelial proliferation. In diabetic retinopathy there is loss of pericytes which leads to formation of microaneurysm, increase in a cellular occluded capillariess and thickening of vascular basement membrane all these abnormalities cause increase in permeability of capillaries which leads swelling of eye (macular edema) and formation of new vessels which proliferates in vitrousl membrane ${ }^{[36,37]}$. Pericyte attach to endothelial cells and activate transforming growth factor-b which causes inhibition of endothelial cells ${ }^{[8]}$ Cause of pericyte loss in diabeties is still unclear one of the hypothesis which was proposed is that pericyticl accumulaton of toxic products like sorbitol or advace glycaton end product ${ }^{[36]} \mathrm{Platelet}$ derived growth factor-b (PGDF-B ) is involve in pericyte recruitment and promotes proliferation and migration of pericytes. PGDF-B deficient mice showes pericyte loss and microaneurysm formation just like in diabetic patient ${ }^{\text {[36]. }}$

Basement membrane:- Thickening of basement membrane of microvasculature of retina due to diabetes is also known as diabetic retinal microangiopathy. Increase in blood glucose level (hyperglycamia) increases flux by polyol, hexosamine pathway, oxidative stress, AGE formation,PKC activation which modify production pattern of autocrine and paracrine by residual and non residual cells in the tissue which impair vessel wall turnover characterized by alter cell and matrix turnover,vascular tone,permiablity,collagin pattern which form surface glycoprotein than normal that increases thickness of basement membrane of retinal microvasculature that causes leakage of blood and proteins in retina causes ischemia and increases VEFG leading to macular edema. Less nutient to tissue increases fibrosis which inturn increases collagen and laminin that are characteristic for basement membrane thickening ${ }^{[38,39]}$.

Clinical Manifestations:- Patients with DM may present in a variety of ways. If the macular edema does not affect the central vision, or fovea, then many patients are asymptomatic. Others may complain of a gradual decrease in vision with distortion. Transient bilateral decrease in vision is also a symptom of DME, and can be caused by acute changes in blood sugars. A sudden increase in floaters in a patient may be a sign of vitreous hemorrhage in a patient with proliferative diabetic retinopathy. Over time, areas of neovascularization that fibrose and regress, may lead to a tractional retinal detachment. This may be gradual or sudden depending on the portion of the eye affected, and usually leaves patients with a "curtain" in their vision or a portion of their visual field missing. Patients with decreased vision and severe pain may be experiencing neovascular glaucoma due to proliferative diabetic retinopathy. Cataracts can also progress over time in diabetic patients. Patients with cataracts often complain of gradual vision loss with glare around lights and decrease in quality of color vision. 
Diagnosis:- Patients with a new diagnosis of diabetes should have a baseline dilated fundus examination by a optometrist or comprehensive ophthalmologist as part of their workup. Patients who are then found to have pathology are referred to a retina specialist for evaluation and possible treatment. In addition to clinical exam, imaging modalities such as fundus photography, optical coherence tomography and fluorescein angiography can aid in the diagnosis of DME. Intravenous Fluorescein Angiography (IVFA) became popular and accepted in the late 60 's. Today, angiography is performed digitally, allowing for real-time evaluation of a patient's retinal circulation. In DR, IVFA can demonstrate both retinal leakage and ischemia, and/or neovascularization especially on wide field angiograms. Angiographic findings in macular edema include progressive leakage from areas of microaneurysmal vascular changes. The most common adverse reactions to occur are nausea (3-5\% of patients), vomiting (7\%) of patients), and pruritus. More severe anaphylactic reactions are rare. Patients may experience temporary erythema and mild pain if dye should extravasate during an injection. This is often relieved with an icepack. Local tissue necrosis has been reported in extremely rare cases. However, more commonly patients experience temporary mild pain and redness, which is often relieved with an icepack. Pregnancy is a relative contraindication for this test ${ }^{[40,41]}$. Optical Coherence Tomography (OCT, is a non-invasive diagnostic tool that obtains in -vivo cross sectional views of the retina. spectral Domain OCT's obtain between 20,000 and 40,000 scans per second, greatly improving resolution, and decreasing likelihood of overlooking pathology ${ }^{[42]}$. Retinal thickness can be measured and compared from visit to visit to the micron, providing an objective means by which to monitor for progression and treatment response. Specifically, OCT helps to quantify and tract intraretinal and subretinal fluid in patients with DME.

\section{Treatment:-}

Management of Diabetic macular edema

Situation 1: Non-center involving macular edema

Situation 2: Non-center involving macular edema with few cystic spaces involving fovea (minimal center involvement)

Situation 3: Center involving macular edema converting to non-center involving macular edema after intravitreal injections.

Situation 4: Predominantly non-center involving macular edema (ring of circinates) after intravitreal injections or laser with plaque of hard exudate and normal foveal contour

Situation 5: Treatment naïve center-involving macular edema

Situation 6: Previously treated center-involving macular edema with multiple Anti-VEGF injections/ Steroid injections/laser

Situation 7: Proliferative diabetic retinopathy with macular edema.

Situation 8: Center involving macular edema with vitreoretinal surface abnormalities

Situation 9: Center involving macular edema in pseudophakic eyes

Situation 10: Macular edema during pregnancy

Situation 11: Macular edema in young type I diabetes

Situation 12: Center involving macular edema in vitrectomized eyes

Situation 13: Ischemic macular edema

Situation 14: Center involving macular edema with history of recent stroke.

Situation 15: Diabetic macular edema in glaucomatous eyes

Situation 16: Considering diabetic macular edema for vitrectomy

Situation 17: Cataract with Diabetic macular edema

Situation 18: Diabetic macular edema with optic nerve abnormalities

Situation 19: Diabetic macular edema with anemia

Situation 20: Diabetic macular edema with mixed retinopathy

\section{Medical Management :-}

The Diabetes Complications and Control Trial (DCCT) and United Kingdom Prospective Diabetes Study (UKPDS) demonstrated the importance of glycemic and blood pressure control in preventing both development and progression of DR in Type 1 and 2 diabetes ${ }^{(47)}$. This remains the best approach for prevention of diabetic retinopathy, and these controls should be prioritized by both the primary care provider as well as the ophthalmologist.

Laser Therapy:- Argon laser photocoagulation is the mainstay of therapy for focal, non-fovea involving diabetic macular edema ${ }^{[48,49]}$. It is performed in the clinical setting as an outpatient procedure. Laser was the standard of 
treatment for DME beginning in the 1980's, and remained the benchmark by which all future treatments were measured until recently. Two landmark studies, the Diabetic Retinopathy Study (DRS) and Early Treatment of Diabetic Retinopathy Study (ETDRS) demonstrated the efficacy of focal laser therapy when applied to non-fovea involving areas of DME. When compared to observation, focal laser resulted in a 50\% reduction in the rate of moderate vision loss at 3 years ${ }^{[51]}$. One of the major benefits of focal laser compared to the other treatment modalities discussed in this review is that it is a noninvasive treatment with patients experiencing minimal, if any discomfort. These spot scan target any leaking microaneurysms and are applied in a grid like fashion overlying areas of thickening. The entire procedure lasts approximately 10-15 minutes, and effects of the laser (decrease in macular edema) are seen approximately 6 weeks afterward. During treatment, care is taken to avoid the foveal center, as laser burns in this location would cause scotomas.

Treatment is recommended for macular edema that meets the criteria for clinical significance, as defined by the ETDRS study ${ }^{[52]}$. The mechanism by which focal laser decreases DME remains unknown, but it is hypothesized that direct photocoagulation of microaneurysms reduces flow and decreases leakage. In addition, laser may stimulate the retinal pigment epithelium, resulting in increased resorption of extracellular fluid ${ }^{[53]}$. Focal laser can be repeated with caution, should recurrences of macular edema occur, and can be performed in conjunction with any of the other treatments for macular edema. While focal laser remains an excellent treatment option for patients with nonfovea involving DME and can prevent further vision loss, it does not necessarily offer the same potential for visual gain associated with intravitreal therapy ${ }^{[54]}$.

Intravitreal Therapy:- Intravitreal therapy is the current gold standard of treatment for cases of focal and diffuse fovea-involving DME. Treatment patterns for DME with intravitreal injections may vary between each retina specialist and even between each patient. Some specialists treat PRN (as needed), and will inject if there is a presence of DME on examination. Others will "load" the patient with a series of 3 monthly injections, followed by a PRN, approach, or a treat and extend approach - during which a patient is treated at each subsequent follow up, but intervals between each follow up gradually increase.

Intravitreal anti-VEGF pathogenesis:-Vascular endothelial growth factor (VEGF) is an essential moderator of bloodretinal barrier breakdown, particularly in regards to angiogenesis and permeability. Hypoxia resulting from

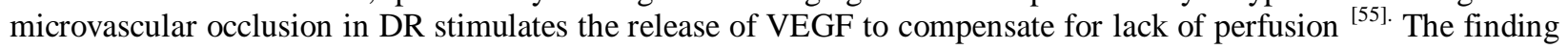
of elevated vitreous VEGF levels in patients with DME led to the proposal of VEGF inhibitors as a potential treatment for DME.

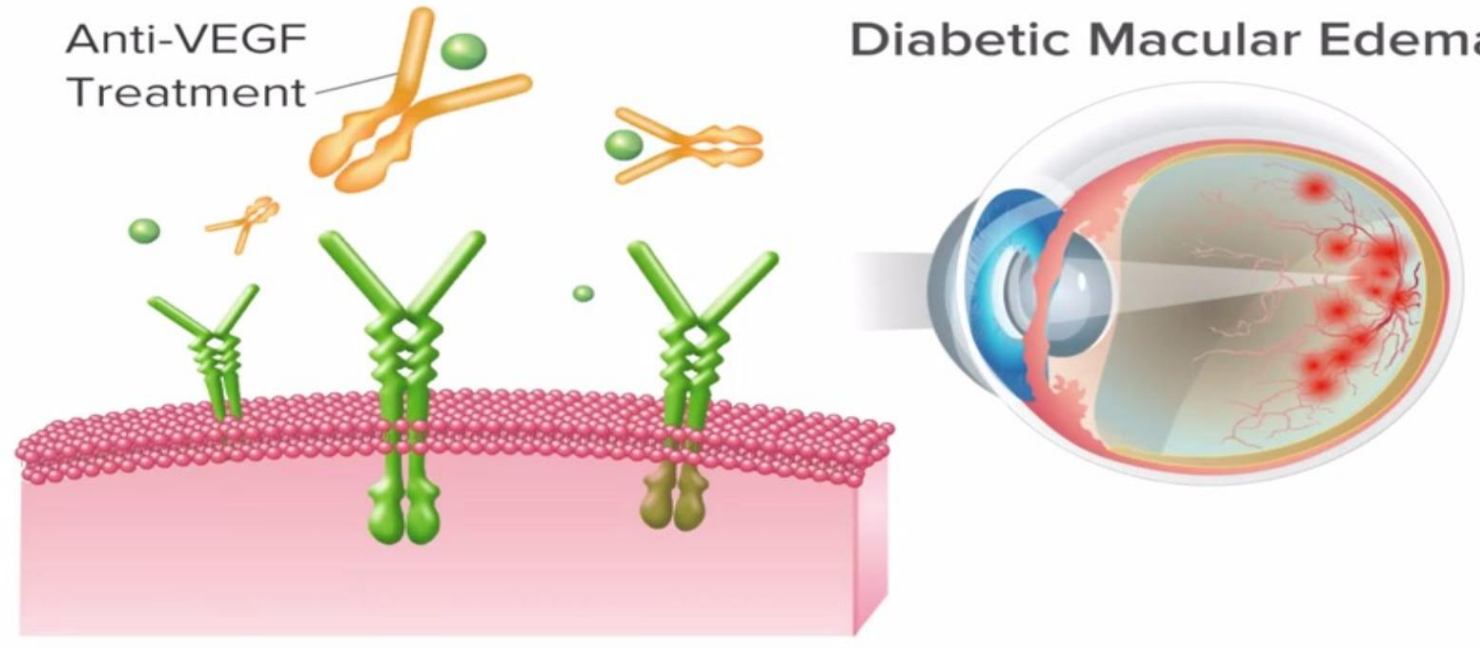

Fig 5:- Anti-VEGF treatment for Diabetic macular edema

\section{Administration Of Medications:-}

Intravitreal injections are administered in the clinical setting. While each retina specialist may have slight differences in terms of preparation for an injection, patients can expect to be in a reclined position, with local anesthesia administered to the treatment eye in the form of drops, gel, or subconjunctival injection. A 5\% betadine wash is performed, and a lid speculum is placed to provide adequate exposure. These measures appear to be 
effective in limiting the incidence of injection - related infection (endophthalmitis), with most studies revealing a $1 / 2000$ risk of this site-threatening complication ${ }^{[56]}$. The intravitreal injection is performed $4 \mathrm{~mm}$ from the limbus conjunctiva) using a 30 -gauge needle on a $1 \mathrm{cc}$ syringe. Approximately $0.05 \mathrm{cc}$ of medication is injected into the center of the vitreous cavity. Patients do not require a patch after the injection. There is no limit to the number of injections a patient can receive, and most patients require multiple injections at intervals as close as 4 weeks.

Bevacizumab:- (Avastin, Genentech/Roche): Bevacizumab is a recombinant humanized monoclonal antibody with an immunogenic Fc fragment that blocks VEGF-A, which was first FDA approved for treatment of metastatic colorectal cancer. It is now widely used off-label for the treatment of DME as well as Age Related Macular Degeneration. Bevacizumab costs approximately $\$ 50$ a dose, much less than its competitor's ranibizumab and aflibercept (priced at \$1800- 2000 a dose). This price difference, as well as the off-label status of intravitreal bevacizumab for DME, has caused a great deal of debate amongst retina specialists regarding which should be used ${ }^{[57]}$. Unfortunately, at this point we have no head-to-head trials comparing the 3 medications for DME, although trials, have found bevacizumab and ranibizumab to have equivalent visual acuity outcomes in the treatment of age related macular degeneration ${ }^{[58,59]}$

Ranibizumab:- (Lucentis, Genentech):-Ranibizumab is a humanized monoclonal antibody fragment that was engineered specifically for ophthalmologic use and was approved by the FDA in 2006 for treatment of age related macular degeneration, and subsequently for DME and retinal vein occlusions., This from systemic circulation quickly, and is thought to have the least systemic absorption when compared to the other antivegf alternatives. Several studies including those by the DRCR net ${ }^{[60]}$, the RESOLVE study ${ }^{[61]}$, and the RISE and RIDE ${ }^{[62]}$ trials confirmed ranibizumab's effectiveness in the treatment of DME, with resulting visual acuity gains.molecule targets VEGF-A with high affinity. Due to its small size and the fact that it is without an Fc fragment, ranibizumab clears from systemic circulation quickly, and is thought to have the least systemic absorption when compared to the other antivegf alternatives. Several studies including those by the DRCR net ${ }^{[60]}$, the RESOLVE study ${ }^{[61]}$, and the RISE and RIDE ${ }^{[62]}$ trials confirmed ranibizumab's effectiveness in the treatment of DME, with resulting visual acuity gains.

Aflibercept:- (Eylea, Regeneron Pharmaceuticals): The most recently developed of the anti VEGF medications, aflibercept is composed of key domains from human VEGF receptors 1 and 2 fused to the Fc domain of human immunoglobulin G1. It's binding affinity to VEGF-A is 100x greater than bevacizumab or ranibizumab ${ }^{[55]}$. Animal studies have shown aflibercept to have a longer intraocular half -life than bevacizumab and ranibizumab, potentially allowing for decreased injection frequency ${ }^{[56]}$. Initially used for exudative age-related macular degeneration, aflibercept is now FDA approved for use in central and branch retinal vein occlusions, and as late as 2014, for use in DME.

Corticosteroids:- Inflammation has also been shown to play a significant role in the development of diabetic macular edema ${ }^{[57]}$. As a result,corticosteroids with both anti-inflammatory and anti-VEGF properties have also been utilized in the treatment of $\mathrm{DME}^{[64]}$. Triamcinolone acetonide can be injected intravitreally in the form of Kenalog (Bristol-Myers Squibb), Triescenc(Alcon), or Trivaris (Allergan). Although Triescence and Trivaris are FDA approved for intravitreal use in treatment of intraocular inflammation, their use for diabetic macular edema remains off label. Ocular side-effects of intravitreal corticosteroid use include cataract formation and intraocular pressure elevation, which occur at significantly greater frequency than with intravitreal anti-VEGF agents ${ }^{[58,59]}$. Because of these concerns, steroids have decreased in popularity, and now are used as a second or third line therapy or in combination with laser or anti-VEGF agent for refractory cases of DME. 


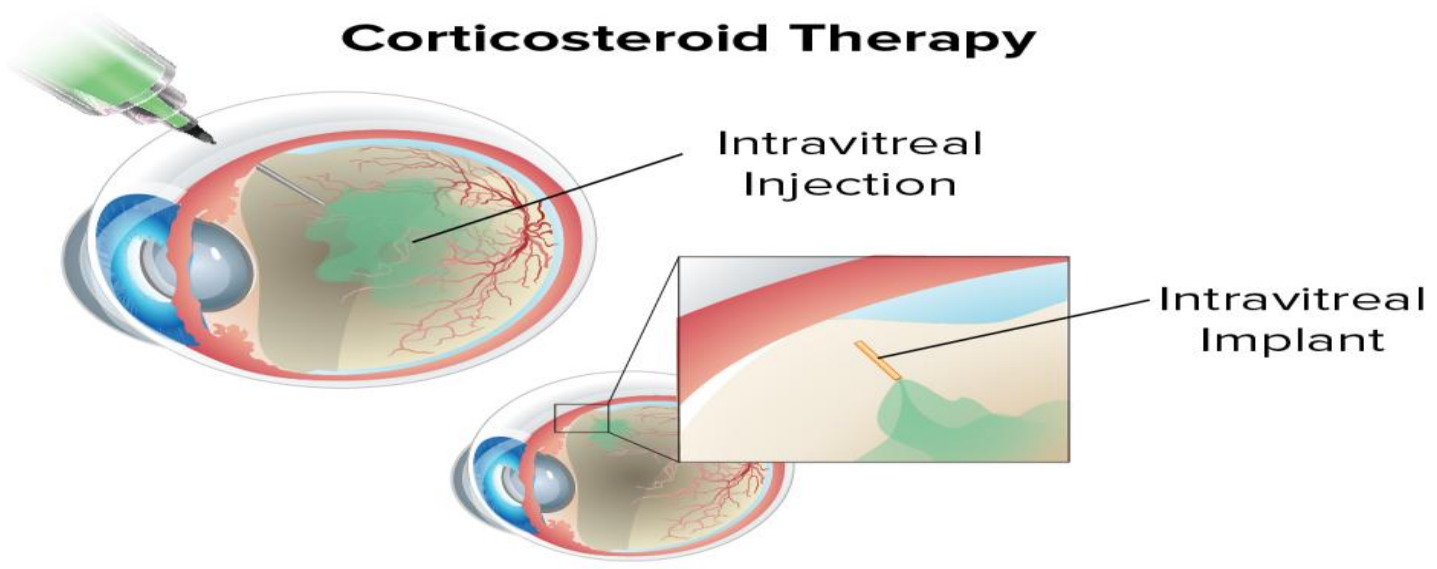

Fig 6:- Corticosteroid therapy for Diabetic Macular Edema

Surgical Treatment:- Traction from interface abnormalities between the vitreous and retina have been considered a potential source of DME, and thus in refractory cases of DME where all other options have been exhausted, pars plana vitrectomy is considered. In addition to relieving vitreomacular traction forces, vitrectomy surgery also increases intraocular oxygen levels and thus may decrease ischemia driven VEGF secretion [58]. Studies of vitrectomy surgery with epiretinal and/or inner limiting membrane peeling have shown decreases in central retinal thickness and in some cases improved visual acuity outcomes ${ }^{[59,60]}$. Though a good option in certain cases, the risk versus benefit of surgery must be considered.

\section{Case Report In Diabetic Macular Edema:-Treatment Approaches For Managing Suboptimal Responses}

\section{Case Report:-}

A 69-year-old woman with a 20-year history of mildly controlled type 2 diabetes (hemoglobin A1c) and hypertension presented for a second opinion regarding the vision in her left eye. Her ocular history was significant for cataract surgery and center-involving DME in the left eye ${ }^{61}$. Despite ongoing monthly injections of bevacizumab (Avastin, Genentech) and ranibizumab (Lucentis, Genentech), and multiple focal macular laser treatments, her visual acuity had stabilized at 20/70 with a significant amount of edema and increased macular lipid (Figure 1A). Four injections of Lucentis had reduced the DME by approximately $20 \%$, after minimal improvement was achieved with six injections of Avastin(Figure 1B).

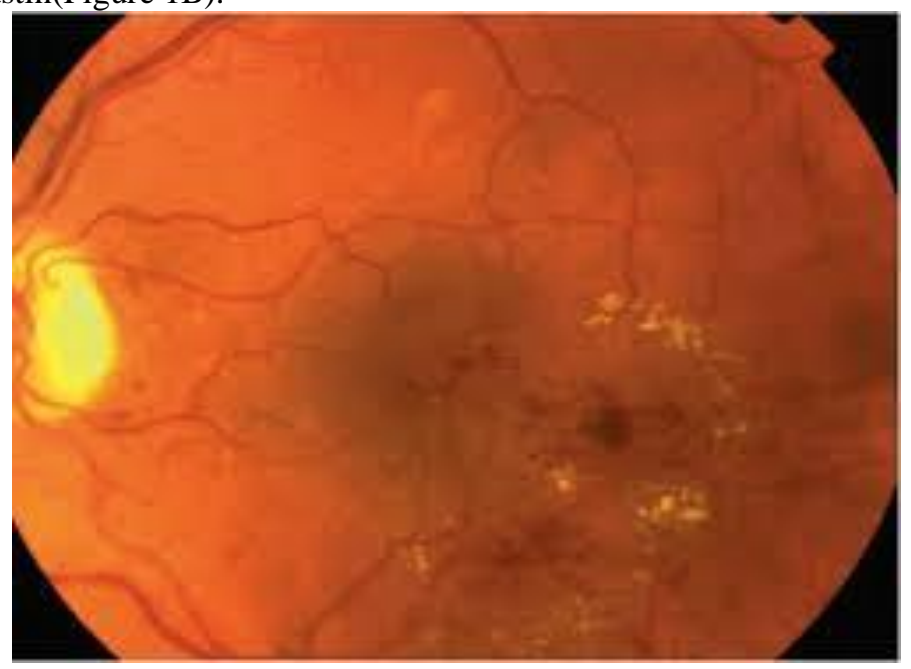

Figure 1A:- After multiple focal macular laser treatments, visual acuity stabilized, but significant edema remained and increased macular lipid was present. 


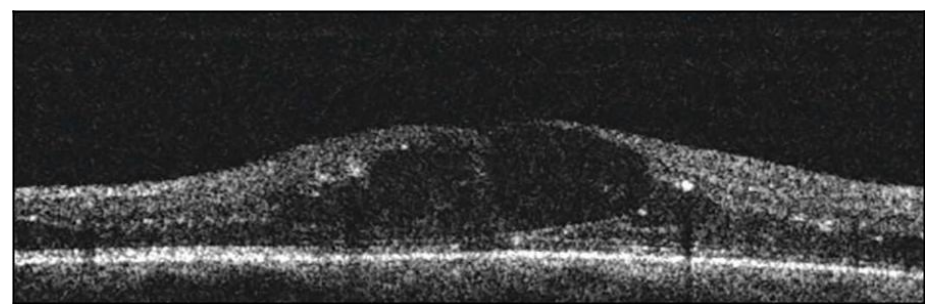

Figure 1B:- The edema was reduced by about $20 \%$ after four injections of Lucentis following six injections of Avastin.

Because of the suboptimal response and the chronicity of the DME, I decided to treat with two concurrent agents ${ }^{62}$ : monthly Lucentis in a modified treat-and-extend protocol, and the dexamethasone intravitreal implant (Ozurdex, Allergan) every 3 months. After 1 month of treatment (agents were given 1 week apart), the edema improved dramatically. After six monthly doses of Lucentis and two every-3-month doses of Ozurdex, the DME and lipid had almost resolved, and visual acuity had improved to 20/30 (Figure 2). The patient received a total of three doses of Ozurdex and one focal macular laser treatment. No significant rise in IOP was noted ${ }^{63-65}$.Currently, 18 months after treatment was initiated, visual acuity remains at 20/30. Te patient is receiving Lucentis every 8 to 10 weeks, without additional steroid for 6 months.

\section{Discussion:-}

Evidence from a recent sub analysis of the Diabetic Retinopathy Clinical Research Network Protocol I study suggests that patients show one of four response types after the first four injections of an anti-VEGF agent, with a quarter of patients being classified as non responders and more than half not having a significant early response. ${ }^{62}$ These data further corroborate findings in subgroups within the Protocol T, VIVID and VISTA, and RISE and RIDE studies, ${ }^{62,67}$ suggesting three categories of DME: mostly VEGF-mediated, minimally VEGF-mediated, and mediated by VEGF and inflammatory processes. Patients in the latter group may benefit significantly from combination therapy with anti-VEGF agents and Corticosteroids. Ozurdex has been shown to be effective in the treatment of persistent DME, DME resistant to anti-VEGF treatment, and DME in difficult to-treat vitrectomized eyes. ${ }^{68}$ The MEAD study further showed that Ozurdex provides robust long-term improvement in visual acuity and macular edema in patients with DME, with a mean of four to five injections over 3 years. Although approximately $40 \%$ of patients in the MEAD study needed IOP-lowering medication ,less than $1 \%$ required incisional glaucoma surgery.

Summary:- For center-involving DME that persists despite anti-VEGF therapy, the durable action of Ozurdex facilitates the use of combination therapy in DME mediated by VEGF and inflammatory processes. In spite of the proven effect of corticosteroids for treating DME, the potential for side effects of cataract and IOP elevation must be monitored. In the future, patients could receive these implants early (after three to four anti-VEGF injections) as additional treatment after a suboptimal response to intravitreal anti-VEGF agents as combination therapy, which may provide some synergistic benefit in certain patients who have both inflammatory and VEGF influences ${ }^{70}$.

\section{Conclusion:-}

As the number of patients diagnosed with diabetes continues to increase, so do the number of patients with macular edema and visual impairment. Monumental advances have been made in the recent past that have given patients and ophthalmologists alike an arsenal of options with which to preserve and improve vision. Even more promising therapeutic options are on the horizon.

\section{References:-}

1. H. King, R.E. Aubert, W.H. Herman, Global burden of diabetes, 1995-2025: prevalence, numerical estimates, and projections. Diabetes Care 21(9), 1414-1431 (1998)

2. L.P. Aiello, T.W. Gardner, G.L. King, G. Blankenship, J.D. Cavallerano, F.L.I.I.I. Ferris, R. Klein, Diabetic retinopathy. Technical review. Diabetes Care 21, 143-156 (1998)

3. Bhagat N, Grigorian RA, Tutela A, Zarbin MA. Diabetic macular edema: pathogenesis and treatment. Surv Ophthalmol. 2009; 54(1):1-32. doi: 10.1016/j.survophthal.2008.10.001.

4. R. Klein, B. Klein, S. Moss, K. Cruickshanks, The Wisconsin Epidemiologic study of Diabetic Retinopathy XV. Ten year incidence and progression of diabetic retinopathy. Arch. Ophthalmol. 112, 1217-1288 (1994) 
5. Conceicaio Lobo, Rui Bernades, J. Faria De Abreu and Jose G. Cunha-AZ,. Nanotechnlogy techniques for Diabetic Macular Edema(DME).

6. Thomas A, M,Armando, Zinman Vol-2008,Pgno:- 112-119.Novel imaging techniques for diabetic macular edema

7. Diabetic macular edema ,Einar Stefansson university of Iceland, National University Hospital, 101 Reykjavi'k, Iceland Received 2 June 2009; accepted 2 June 2009 Available online 5 August 2009

8. Guyton, Arthur; Hall, John (2006). "Chapter 16: The Microcirculation and the Lymphatic System". In Gruliow, Rebecca. Textbook of Medical Physiology (Book) (11th ed.). Philadelphia, Pennsylvania: Elsevier Inc. pp. $187-188$.

9. Thomas A. Ciulla, M,Armando G. Amador, MD,Ernard Zinman, MDCM, FRCP(C), FACP3, Diabetic Retinopathy and Diabetic Macular Edema Pathophysiology, screening, and novel therapies

10. E. Aaron Runkle, David A. Antonett The Blood-Retinal Barrier: Structure and Functional Significance

11. J. G. Cunha-Vaz, The blood-retinal barriers, October 1976, Volume 41, Issue 2, pp 287-327

12. http://www.blueconemonochromacy.org/how-the-eye-functions/

13. Endothelial adherens and tight junctions in vascular homeostasis, inflammation and angiogenesis Yann Wallez 1 , Philippe Huber * Received 25 May 2007; received in revised form 3 September 2007; accepted 5 September 2007 Available online 15 September 2007

14. Endothelial Cell-to-Cell Junctions: Molecular Organization and Role in Vascular Homeostasis Gianfranco Bazzoni and Elisabetta Dejana

15. T. Hirase, J.M. Staddon, M. Saitou, Y. Ando-Akatsuka, M. Itoh, M. Furuse, K. Fujimoto, S. Tsukita, L.L. Rubin, Occludin as a possible determinant of tight junction permeability in endothelial cells, J. Cell Sci. 110 (Pt 14) (1997) 1603-1613.

16. S. Hori, S. Ohtsuki, K. Hosoya, E. Nakashima, T. Terasaki, A pericytederived angiopoietin-1 multimeric complex induces occludin gene expression in brain capillary endothelial cells through Tie-2 activation in vitro, J. Neurochem. 89 (2004) 503-513

17. D.A. Antonetti, A.J. Barber, S. Khin, E. Lieth, J.M. Tarbell, T.W. Gardner, Vascular permeability in experimental diabetes is associated with reduced endothelial occludin content: vascular endothelial growth factor decreases occludin in retinal endothelial cells. Penn State Retina Research Group, Diabetes 47 (1998) 1953-1959

18. M.A. Behzadian, L.J. Windsor, N. Ghaly, G. Liou, N.T. Tsai, R.B. Caldwell, VEGF-induced paracellular permeability in cultured endothelial cells involves urokinase and its receptor, FASEB J. 17 (2003) 752-754.

19. González-Mariscal L, Betanzos A, Nava P, Jaramillo BE: Tight junction proteins. Prog Biophys Mol Biol 2003, 81:1-44.

20. Saitou M, Furuse MS H, Schulzke JD, Fromm M, Takano H, Noda T, Tsukita S: Complex phenotype of mice lacking occludin, a component of tight junction strands. Mol Biol Cell 2000, 11:4131-4142.

21. Mary Gregory DGC: Identification of multiple claudins in the rat epididymis. Mol Reprod Dev 2006, 73:580588.

22. Hou J, Gomes AS, Paul DL, Goodenough DA: Study of claudin function by RNA interference. J Biol Chem 2006, 281:36117-36123.

23. Morris AP, Tawil A, Berkova Z, Wible L, Smith CW, Cunningham SA: Junctional Adhesion Molecules (JAMs) are differentially expressed in fibroblasts and co-localize with ZO-1 to adherens-like junctions. Cell Commun Adhes 2006, 13:233-247.

24. Ebnet K, Suzuki A, Ohno S, Vestweber D: Junctional adhesion molecules (JAMs): more molecules with dual functions? J Cell Sci 2004, 117:19-29.

25. Weber C, Fraemohs L, Dejana E: The role of junctional adhesion molecules in vascular inflammation. Nat Rev Immunol 2007, 7:467-477.

26. What is VEGF By Dr Ananya Mandal, MD

27. Ferrara, N. (1999) Curr. Top. Microbiol. Immunol. 237:1.

28. Houck, K.A. et al. (1992) J. Biol. Chem. 267:26031, (1991) Mol. Endocrinol. 5:1806.

29. Dvorak, H.F. et al. (1999) Curr. Top. Microbiol. Immunol. 237:97.

30. Yang, R. et al. (1996) J. Cardiovasc. Pharmacol. 27:838.

31. Diabetic Retinopathy and VEGF N. Gupta1, S. Mansoor1,2, A. Sharma1,3, A. Sapkal1, J. Sheth3, P. Falatoonzadeh1 , B.D. Kuppermann1 and M.C. Kenney

32. Bates DO, Cui TG, Doughty JM, Winkler M, Sugiono M, Shields JD, Peat D, Gillatt D, Harper SJ: VEGF165b, an inhibitory splice variant of vascular endothelial growth factor, is down-regulated in renal cell carcinoma. Cancer Res 2002, 62:4123-4131. 
33. Perrin RM, Konopatskaya O, Qiu Y, Harper S, Bates DO, Churchill AJ:Diabetic retinopathy is associated with a switch in splicing from anti- to pro-angiogenic isoforms of vascular endothelial growth factor.Diabetologia 2005, 48:2422-2427.

34. Cebe Suarez AZ, FaKB H: The role of VEGF receptors in angiogenesis;complex partnerships. Cell Mol Life Sci 2006, 63:601-615.

35. Mechanisms of Macular Edema and Therapeutic Approaches Antonia M. Joussen, Thomas J. Wolfensberger

36. Vascular Endothelial Growth Factor (VEGF) Updated April 9, 2014 Published November 19, 2009

37. Hammes, H.-P.; Lin, J.; Renner, O.; Shani, M.; Lundqvist, A.; Betsholtz, C.; Brownlee, M.; Deutsch, U. (2002). "Pericytes and the Pathogenesis of Diabetic Retinopathy". Diabetes. 51 (10): 3107-12.

38. Bergers, G.; Song, S (2005). "The role of pericytes in blood-vessel formation and maintenance". NeuroOncology. 7 (4): 452-64. doi:10.1215/S1152851705000232. PMC 1871727 PMID 16212810

39. Pathogenetic mechanisms of diabetic microangiopathy Umberto Di Mario, Giuseppe Pugliese

40. Microangiopathy From Wikipedia, the free encyclopedia.

41. Gass JD, Sever RJ, Sparks D, Goren J. A combined technique of fluorescein funduscopy and angiography of the eye. Arch Ophthalmol. 1967; 78(4):455-461.

42. Yannuzzi LA, Rohrer KT, Tindel LJ, Sobel RS, Costanza MA, Shields W, et al. Fluorescein angiography complication survey. Ophthalmology. 1986; 93(5):611-617.

43. Huang D, Swanson EA, Lin CP, Schuman JS, Stinson WG, Chang W, et al. Optical coherence tomography. Science. 1991; 254(5035):1178-1181

44. Robert Turner, Rury Holman, Irene Stratton, Carole Cull, Valeria Frighi, Susan Manley, et al. Tight blood pressure control and risk of macrovascular and microvascular complications in type 2 diabetes: UKPDS 38. UK Prospective Diabetes Study Group. Bmj. 1998; 317(7160):703-713.

45. Blankenship GW. Diabetic macular edema and argon laser photocoagulation: a prospective randomized study. Ophthalmology. 1979; 86(1):69-78.

46. Writing Committee for the Diabetic Retinopathy Clinical Research Network. Fong DS, Strauber SF, Aiello LP, Beck RW, Callanan DG, Danis RP, et al. Comparison of the modified Early Treatment Diabetic Retinopathy Study and mild macular grid laser photocoagulation strategies for diabetic macular edema. Arch Ophthalmol. 2007; 125(4):469-480.

47. Photocoagulation for Diabetic Macular Edema. Early Treatment Diabetic Retinopathy Study report number 1. Early Treatment Diabetic Retinopathy Study research group. Arch Ophthalmol. 1985; 103(12):1796-1806. doi:10.1001/archopht.1985.

48. Lee CM, Olk RJ. Modified grid laser photocoagulation for diffuse diabetic macular edema. Long-term visual results. Ophthalmology. 1991; 98(10):1594-1602

49. Funatsu H, Yamashita H, Noma H, Mimura T, Yamashita T, Hori S. Increased levels of vascular endothelial growth factor and interleukin-6 in the aqueous humor of diabetics with macular edema. Am J Ophthalmol. 2002; 133(1):70-77.

50. Dakin HA, Wordsworth S, Rogers CA, Abangma G, Raftery J, Harding SP, et al. Cost-effectiveness of ranibizumab and bevacizumab for age-related macular degeneration: 2-year findings from the IVAN randomised trial. BMJ open. 2014; 4(7):e005094. doi:10.1136/ bmjopen-2014-005094.

51. Papadopoulos N, Martin J, Ruan Q, Rafique A, Rosconi MP, Shi E, et al. Binding and neutralization of vascular endothelial growth factor (VEGF) and related ligands by VEGF Trap, ranibizumab and bevacizumab. Angiogenesis. 2012; 15(2):171-85. doi: 10.1007/ s10456-011-9249-6.

52. Do DV, Schmidt-Erfurth U, Gonzalez VH, Gordon CM, Tolentino M, Berliner AJ, et al. The DA VINCI Study: phase 2 primary results of VEGF Trap-Eye in patients with diabetic macular edema. Ophthalmology. 2011; 118(9):1819-1826. doi: 10.1016/j.ophtha.2011.02.018.

53. Diabetic Retinopathy Clinical Research N, Scott IU, Edwards AR, Beck RW, Bressler NM, Chan CK, Elman $\mathrm{MJ}$, et al. A phase II randomized clinical trial of intravitreal bevacizumab for diabetic macular edema. Ophthalmology. 2007; 114(10):1860-1867.

54. Massin P, Bandello F, Garweg JG, Hansen LL, Harding SP, Larsen M, et al. Safety and efficacy of ranibizumab in diabetic macular edema (RESOLVE Study): a 12-month, randomized, controlled, doublemasked, multicenter phase II study. Diabetes care. 2010; 33(11):2399- 2405. doi: 10.2337/dc10-0493.

55. Nguyen QD, Brown DM, Marcus DM, Boyer DS, Patel S, Feiner L, et al. Ranibizumab for diabetic macular edema: results from 2 phase III randomized trials: RISE and RIDE. Ophthalmology. 2012 Apr;119(4):789-801. doi: 10.1016/j.ophtha.2011.12.039. 
56. Funatsu H, Noma H, Mimura T, Eguchi S, Hori S. Association of vitreous inflammatory factors with diabetic macular edema. Ophthalmology. 2009; 116(1):73-9. doi: 10.1016/j.ophtha.2008.09.037. 21.Nauck M, Karakiulakis G, Perruchoud AP, Papakonstantinou E, Roth M. Corticosteroids inhibit the expression of the vascular endothelial growth factor gene in human vascular smooth muscle cells. Eur J Pharmacol. 1998; 341(23):309-315.

57. Bandello F, Preziosa C, Querques G, Lattanzio R. Update of intravitreal steroids for the treatment of diabetic macular edema. Ophthalmic Res. 2014; 52(2):89-96. doi: 10.1159/000362764.

58. Zacharias LC, Lin T, Migon R, Ghosn C, Orilla W, Feldmann B, et al. Assessment of the differences in pharmacokinetics and pharmacodynamics between four distinct formulations of triamcinolone acetonide. Retina. 2013 Mar;33(3):522-31. doi: 10.1097/IAE.0b013e3182647f69.

59. Holekamp NM, Shui YB, Beebe DC. Vitrectomy surgery increases oxygen exposure to the lens: a possible mechanism for nuclear cataract formation. Am J Ophthalmol. 2005 Feb;139(2):302-310.

60. Diabetic Retinopathy Clinical Research Network Writing C, Haller JA, Qin H, Apte RS, Beck RR, Bressler NM, et al. Vitrectomy outcomes in eyes with diabetic macular edema and vitreomacular traction. Ophthalmology. 2010; 117(6):1087-1093.e3. doi: 10.1016/j. ophtha.2009.10.040.

61. Bonnin S, Sandali O, Bonnel S, Monin C, El Sanharawi M. Vitrectomy with internal limiting membrane peeling for tractional and nontractional diabetic macular edema Long-term Results of a Comparative Study. Retina. 2015; 35(5):921-928. doi: 10.1097/IAE.0000000000000433.

62. Nguyen QD, Brown DM, Marcus DM, et al; RISE and RIDE Research Group. Ranibizumab for diabetic macular edema: results from 2 phase III randomized trials: RISE and RIDE. Ophthalmology. 2012;119:789801 .

63. Brown DM, Nguyen QD, Marcus DM, et al; RIDE and RISE Research Group. Long-term outcomes of ranibizumab therapy for diabetic macular edema: the 36-month results from two phase III trials: RISE and RIDE. Ophthalmology. 2013;120:2013-2022.

64. Funatsu H, Noma H, Mimura T, Eguchi S, Hori S. Association of vitreous inflammatory factors with diabetic macular edema. Ophthalmology. 2009;116:73-79.

65. Gillies MC, Lim LL, Campain A, et al. A randomized clinical trial of intravitreal bevacizumab versus intravitreal dexamethasone for diabetic macular edema: the BEVORDEX study. Ophthalmology. 2014;121:2473-2481.

66. Boyer DS, Yoon YH, Belfort R, Jr., et al. Three-year, randomized, sham-controlled trial of dexamethasone intravitreal implant in patients with diabetic macular edema. Ophthalmology. 2014;121:1904-1914.

67. Bressler SB, Qin H, Beck RW, et al; Diabetic Retinopathy Clinical Research Network. Factors associated with changes in visual acuity and OCT thickness at 1 year after treatment for diabetic macular edema with ranibizumab. Arch Ophthalmol. 2012;30:1153-1161.

68. Korobelnik JF, Do DV, Schmidt-Erfurth U, et al. Intravitreal aflibercept for diabetic macular edema. Ophthalmology. 2014;121:2247-2254.

69. Pacella E, Vestri AR, Muscella R, et al. Preliminary results of an intravitreal dexamethasone implant (Ozurdex) in patients with persistent diabetic macular edema. Clin Ophthalmol. 2013;7:1423-1428.

70. Lazic R, Lukic M, Boras I, et al. Treatment of anti-vascular endothelial growth factor-resistant diabetic macular edema with dexamethasone intravitreal implant. Retina. 2014;34:719-724.

71. Boyer DS, Faber D, Gupta S, et al; Ozurdex CHAMPLAIN Study Group. Dexamethasone intravitreal implant for treatment of diabetic macular edema in vitrectomized patients. Retina. 2011;31:915-923. 